\title{
THE INFRARED PROPERTIES OF SOURCES MATCHED IN THE WISE ALL-SKY AND HERSCHEL ATLAS SURVEYS
}

\author{
Nicholas A. Bond ${ }^{1}$, Dominic J. Benford ${ }^{1}$, Jonathan P. Gardner ${ }^{1}$, Alexandre Amblard ${ }^{2}$, Simone Fleuren $^{3}$, \\ Andrew W. Blain ${ }^{4}$, Loretta Dunne ${ }^{5}$, Daniel J. B. Smith ${ }^{6}$, Steve J. Maddox ${ }^{5}$, Carlos Hoyos ${ }^{5}$, Maarten Baes ${ }^{7}$, \\ David Bonfield ${ }^{6}$, Nathan Bourne $^{5}$, Carrie Bridge ${ }^{8}$, Sara Buttiglione ${ }^{9}$, Antonio Cava ${ }^{10}$, David Clements ${ }^{11}$, \\ Asantha Cooray ${ }^{12}$, Ali Dariush ${ }^{13}$, Gianfranco de Zotti ${ }^{9,14}$, Simon Driver ${ }^{15,16}$, Simon Dye ${ }^{5}$, Steve Eales ${ }^{17}$, \\ Peter Eisenhardt ${ }^{18}$, Rosalind Hopwood ${ }^{13}$, Edo Ibar ${ }^{19}$, Rob J. Ivison ${ }^{19}$, Matt J. Jarvis ${ }^{6,20}$, Lee Kelvin ${ }^{15,16}$, \\ aaron S. G. Robotham ${ }^{15,16}$, Pasquale Temi ${ }^{2}$, Mark Thompson ${ }^{6}$, ChaO-Wei Tsai $^{21}$, Paul van der Werf $^{22}$, \\ EDWARD L. WRIGHT ${ }^{23}$, JiNGWEN Wu ${ }^{16}$, AND LIN YAN ${ }^{24}$ \\ ${ }^{1}$ Cosmology Laboratory (Code 665), NASA Goddard Space Flight Center, Greenbelt, MD 20771, USA \\ 2 Astrophysics Branch, NASA/Ames Research Center, MS 245-6, Moffett Field, CA 94035, USA \\ ${ }^{3}$ School of Mathematical Sciences, Queen Mary, University of London, Mile End Road, London E1 4NS, UK \\ ${ }^{4}$ Department of Physics \& Astronomy, University of Leicester, University Road, Leicester LE1 7RH, UK \\ ${ }^{5}$ School of Physics and Astronomy, University of Nottingham, University Park, Nottingham NG7 2RD, UK \\ ${ }^{6}$ Centre for Astrophysics Research, Science \& Technology Research Institute, University of Hertfordshire, Hatfield, Herts AL10 9AB, UK \\ ${ }^{7}$ Sterrenkundig Observatorium, Universiteit Gent, Krijgslaan 281 S9, B-9000 Gent, Belgium \\ ${ }^{8}$ Division of Physics, Mathematics, and Astronomy, California Institute of Technology, Pasadena, CA 91125, USA \\ 9 INAF-Osservatorio Astronomico di Padova, Vicolo Osservatorio 5, I-35122 Padova, Italy \\ ${ }^{10}$ Departamento de Astrofísica, Facultad de CC. Físicas, Universidad Complutense de Madrid, E-28040 Madrid, Spain \\ ${ }^{11}$ Imperial College, Astrophysics Group, Blackett Lab, Prince Consort Road, London SW7 2AZ, UK \\ ${ }_{12}$ Department of Physics \& Astronomy, University of California, Irvine, CA 92697, USA \\ ${ }^{13}$ Physics Department, Imperial College London, South Kensington Campus, SW7 2AZ, UK \\ ${ }^{14}$ SISSA, Via Bonomea 265, I-34136 Trieste, Italy \\ ${ }^{15}$ International Centre for Radio Astronomy Research (ICRAR), University of Western Australia, Crawley WA 6009, Australia \\ ${ }^{16}$ SUPA, School of Physics and Astronomy, University of St. Andrews, North Haugh, St. Andrews KY169SS, UK \\ ${ }^{17}$ School of Physics and Astronomy, Cardiff University, The Parade, Cardiff CF24 3AA, UK \\ ${ }^{18}$ Jet Propulsion Laboratory, California Institute of Technology, Pasadena, CA 91109, USA \\ ${ }^{19}$ UK Astronomy Technology Centre, Royal Observatory, Blackford Hill, Edinburgh EH9 3HJ, UK \\ ${ }^{20}$ Physics Department, University of the Western Cape, Cape Town 7535, South Africa \\ ${ }^{21}$ IPAC, California Institute of Technology, Pasadena, CA 91125, USA \\ ${ }^{22}$ Leiden Observatory, Leiden University, P.O. Box 9513, 2300 RA Leiden, The Netherlands \\ ${ }^{23}$ UCLA Astronomy, P.O. Box 951547, Los Angeles, CA 90095-1547, USA \\ ${ }^{24}$ Spitzer Science Center, California Institute of Technology, 1200 E. California Blvd., Pasadena, CA 91125, USA \\ Received 2011 November 28; accepted 2012 March 21; published 2012 April 12
}

\begin{abstract}
We describe the infrared properties of sources detected over $\sim 36 \mathrm{deg}^{2}$ of sky in the GAMA $15 \mathrm{hr}$ equatorial field, using data from both the Herschel Astrophysical Terahertz Large-Area Survey (H-ATLAS) and Wide-field Infrared Survey (WISE). With $5 \sigma$ point-source depths of 34 and $0.048 \mathrm{mJy}$ at $250 \mu \mathrm{m}$ and $3.4 \mu \mathrm{m}$, respectively, we are able to identify $50.6 \%$ of the H-ATLAS sources in the WISE survey, corresponding to a surface density of $\sim 630 \mathrm{deg}^{-2}$. Approximately two-thirds of these sources have measured spectroscopic or optical/near-IR photometric redshifts of $z<1$. For sources with spectroscopic redshifts at $z<0.3$, we find a linear correlation between the infrared luminosity at $3.4 \mu \mathrm{m}$ and that at $250 \mu \mathrm{m}$, with $\pm 50 \%$ scatter over $\sim 1.5$ orders of magnitude in luminosity, $\sim 10^{9}-10^{10.5} L_{\odot}$. By contrast, the matched sources without previously measured redshifts $(r \gtrsim 20.5)$ have $250-350 \mu \mathrm{m}$ flux density ratios which suggest either high-redshift galaxies $(z \gtrsim 1.5)$ or optically faint low-redshift galaxies with unusually low temperatures $(T \lesssim 20)$. Their small $3.4-250 \mu \mathrm{m}$ flux ratios favor a highredshift galaxy population, as only the most actively star-forming galaxies at low redshift (e.g., Arp 220) exhibit comparable flux density ratios. Furthermore, we find a relatively large active galactic nucleus fraction $(\sim 30 \%)$ in a $12 \mu \mathrm{m}$ flux-limited subsample of H-ATLAS sources, also consistent with there being a significant population of high-redshift sources in the no-redshift sample.
\end{abstract}

Key words: cosmology: observations - galaxies: general - galaxies: high-redshift - galaxies: statistics - infrared: galaxies - surveys

\section{INTRODUCTION}

The advent of submillimeter astronomy has opened a new window into the universe, allowing us to probe dusty starforming galaxies at high redshift, as well as cold dust in nearby galaxies. Along with the recent success of the balloon-borne BLAST experiment (Pascale et al. 2008; Eales et al. 2009), the launch of the Herschel Space Observatory ${ }^{25}$ (Pilbratt et al. 2010) allows us to probe wavelengths from 55 to $672 \mu \mathrm{m}$ to a spatial resolution of $\lesssim 10^{\prime \prime}$. Its largest open-time key project, the Herschel Astrophysical Terahertz Large-Area Survey

\footnotetext{
25 Herschel is an ESA space observatory with science instruments provided by European-led Principal Investigator consortia and with important participation from NASA.
} 
(H-ATLAS; Eales et al. 2010a), will observe $\sim 550 \mathrm{deg}^{2}$ of sky and detect more than 300,000 galaxies.

Based upon a preliminary cross-identification with the Galaxy and Mass Assembly survey (GAMA; Driver et al. 2011) and the Sloan Digital Sky Survey (SDSS; Abazajian et al. 2009), 50\% of the sources detected in the H-ATLAS survey are at $z<1$ (Smith et al. 2011). In addition, an analysis of the FIR colors of the remaining H-ATLAS sources with flux densities above $35 \mathrm{mJy}$ at $350 \mu \mathrm{m}$ and $>3 \sigma$ detections at $250 \mu \mathrm{m}$ and $500 \mu \mathrm{m}$ suggests an average redshift of $z \sim 2$ (Amblard et al. 2010; Lapi et al. 2011).

Another method of constraining the redshift distribution of FIR-selected sources is to look for counterparts in the nearand mid-infrared (NIR and MIR). S. Fleuren et al. (2012) have performed source matching to survey data from the VISTA Kilo-degree Infrared Galaxy survey (VIKING; W. Sutherland et al., in preparation), but these data only extend to $\sim 2 \mu \mathrm{m}$. Starting in 2009 December, the Wide-field Infrared Survey Explorer $^{26}$ (WISE; Wright et al. 2010) began its mission to observe the entire sky in four bands, ranging from 3.4 to $22 \mu \mathrm{m}$, at $\lesssim 12^{\prime \prime}$ resolution. Following the first public data release in 2011 April, 24,000 $\mathrm{deg}^{2}$ of IR images and source catalogs became available to the public, including $36 \mathrm{deg}^{2}$ of sky in the equatorial plane covered by the H-ATLAS survey.

The purpose of this Letter is to describe the infrared properties of identified WISE counterparts to H-ATLAS sources within the GAMA $15 \mathrm{hr}$ field (G15). Throughout, we will assume a concordance cosmology with $H_{0}=71 \mathrm{~km} \mathrm{~s}^{-1} \mathrm{Mpc}^{-1}$, $\Omega_{\mathrm{m}}=0.27$, and $\Omega_{\Lambda}=0.73$ (Spergel et al. 2007). In a subsequent paper, we will report WISE cross-identifications over the entire H-ATLAS area and perform spectral energy distribution (SED) fits to the matched sources.

\section{DATA AND METHODOLOGY}

Herschel observations of the G15 field include imaging data at 250,350 , and $500 \mu \mathrm{m}$ from the SPIRE instrument, with respective beam FWHM of 18.'1, 25".2, and 36".6 (Griffin et al. 2010), as well as 100 and $160 \mu \mathrm{m}$ imaging from the PACS instrument (Poglitsch et al. 2010). The field subtends approximately $12^{\circ}$ in right ascension and $3^{\circ}$ in declination and is centered on the equatorial plane at $\alpha=14^{\mathrm{h}} 30^{\mathrm{m}}$. The H-ATLAS G15 source catalog (L. Dunne et al., in preparation) is constructed using the same method as the Science Demonstration Phase catalog (Rigby et al. 2011). It contains 27,481 sources detected at $>5 \sigma$ in any of the three SPIRE bands and reaches point-source depths of 34,40 , and $44 \mathrm{mJy}$ at 250,350 , and $500 \mu \mathrm{m}$, respectively.

The WISE first public data release contains four-band coverage of the entire G15 field to $5 \sigma$ point-source depths of 0.048 , $0.10,0.73$, and $5.9 \mathrm{mJy}$ at $3.4,4.6,12$, and $22 \mu \mathrm{m}$. The angular resolution in these bands is $6^{\prime \prime} .1,6.4,6.5$, and $12^{\prime \prime} .0$, respectively (Wright et al. 2010). There are $\sim 240,000$ WISE sources within G15 detected at $>7 \sigma$ in at least one of the four WISE bands, corresponding to $\sim 0.14$ WISE sources per $10^{\prime \prime}$ radius aperture. In the WISE preliminary release catalog, close pairs of sources are not deblended for separations $\lesssim 9^{\prime \prime}$, so when a background or foreground source appears near an H-ATLAS source position, it will often be blended with the true counterpart.

In addition to the infrared data from WISE and H-ATLAS, the G15 region has spectroscopic redshifts from the GAMA

\footnotetext{
26 For a description of the WISE mission, see http://wise2.ipac.caltech.edu/docs/release/prelim/expsup/
}

survey ( $r \lesssim 19.4$, Driver et al. 2011), and photometric redshifts obtained using optical/NIR photometry from SDSS, VIKING, and the UK Infrared Deep Sky Survey Large Area Survey (UKIDSS-LAS; Lawrence et al. 2007). These photometric redshifts are derived following Smith et al. (2011) and have typical redshift uncertainties of $\sigma_{z} / z \sim 0.15$. Of the 27,481 $\mathrm{H}$-ATLAS sources in the region, $16 \%$ have reliable spectroscopic redshifts and $39 \%$ have photometric redshifts.

As a result of the high space density of $3.4 \mu \mathrm{m}$ sources, a naive matching to the H-ATLAS source positions will result in a non-negligible number of misidentifications. A significant fraction of these misidentifications will be foreground stars that are blue in all WISE bands and undetectable in H-ATLAS, so we can reduce the contamination rate of our matched catalog by considering only WISE sources with [3.4] - [4.6] $>0$. The magnitude distribution of all background/foreground WISE sources is such that $\sim 20 \%$ are bluer than this limit, as compared to $<1 \%$ of sources within $10^{\prime \prime}$ of an H-ATLAS position.

To estimate the fraction of H-ATLAS sources with detectable counterparts in the remainder of the WISE source catalog, we use the method of S. Fleuren et al., in preparation, where the detection rate is given by

$$
Q_{0}=1-\frac{\bar{S}}{\bar{B}},
$$

where $\bar{S}$ is the fraction of unmatched positions in the H-ATLAS catalog and $\bar{B}$ is the fraction of unmatched random positions. Matching all WISE sources within 10" (within which we expect $>99 \%$ of the true matches to lie) and using $10^{5}$ random positions within the G15 field, we find $Q_{0}=0.632 \pm 0.004$. This is larger than the value found in the SDSS galaxy catalog $\left(Q_{0}=0.583\right.$, Smith et al. 2011), but smaller than in the VIKING $K s$ band ( $Q_{0}=0.75 ;$ S. Fleuren et al., in preparation). By contrast, we find $Q_{0}=0.012 \pm 0.002$ within the subset of WISE sources with $[3.4]-[4.6]<0$, suggesting that this color cut was effective in removing stars.

In Figure 1, we use Equation (1) to estimate the IR detection rate as a function of FIR flux density. For sources brighter than $100 \mathrm{mJy}$ at $250 \mu \mathrm{m}$, we find a WISE3.4 $\mu \mathrm{m}$ counterpart $>95 \%$ of the time. The majority of these sources $(78 \%)$ have measured spectroscopic or photometric redshifts of $<0.5$, suggesting that the low-redshift mode of star-forming galaxies dominates submillimeter sources above this flux density. Below this FIR flux density, the NIR and MIR detection rates drop rapidly as the high-redshift mode accounts for an increasing fraction of the submillimeter sources (see Figure 2).

Approximately $1 \%$ of the objects in our $250 \mu \mathrm{m}$ selected sample (Negrello et al. 2007), including $\sim 50 \%$ of sources brighter than $100 \mathrm{mJy}$ at $500 \mu \mathrm{m}$ (Negrello et al. 2010; Hopwood et al. 2011), are expected to be strongly lensed $z>1$ galaxies. Of the 46 such sources in the G15 region, $\sim 20 \%$ are undetected at 12 and $22 \mu \mathrm{m}$, most likely because the $500 \mu \mathrm{m}$ bandpass shifts higher in the blackbody curve at high redshift, while the rest-frame MIR flux density declines blueward of $22 \mu \mathrm{m}$ (e.g., Rieke et al. 2009).

The identification of individual NIR and MIR counterparts is more subtle, as we want to eliminate as many of the false matches as possible. Here, we use the likelihood ratio technique of Sutherland \& Saunders (1992), which was implemented for the H-ATLAS survey in Smith et al. (2011, hereafter S11). The likelihood that a given WISE source is a counterpart to an H-ATLAS source is a function of the radial probability 

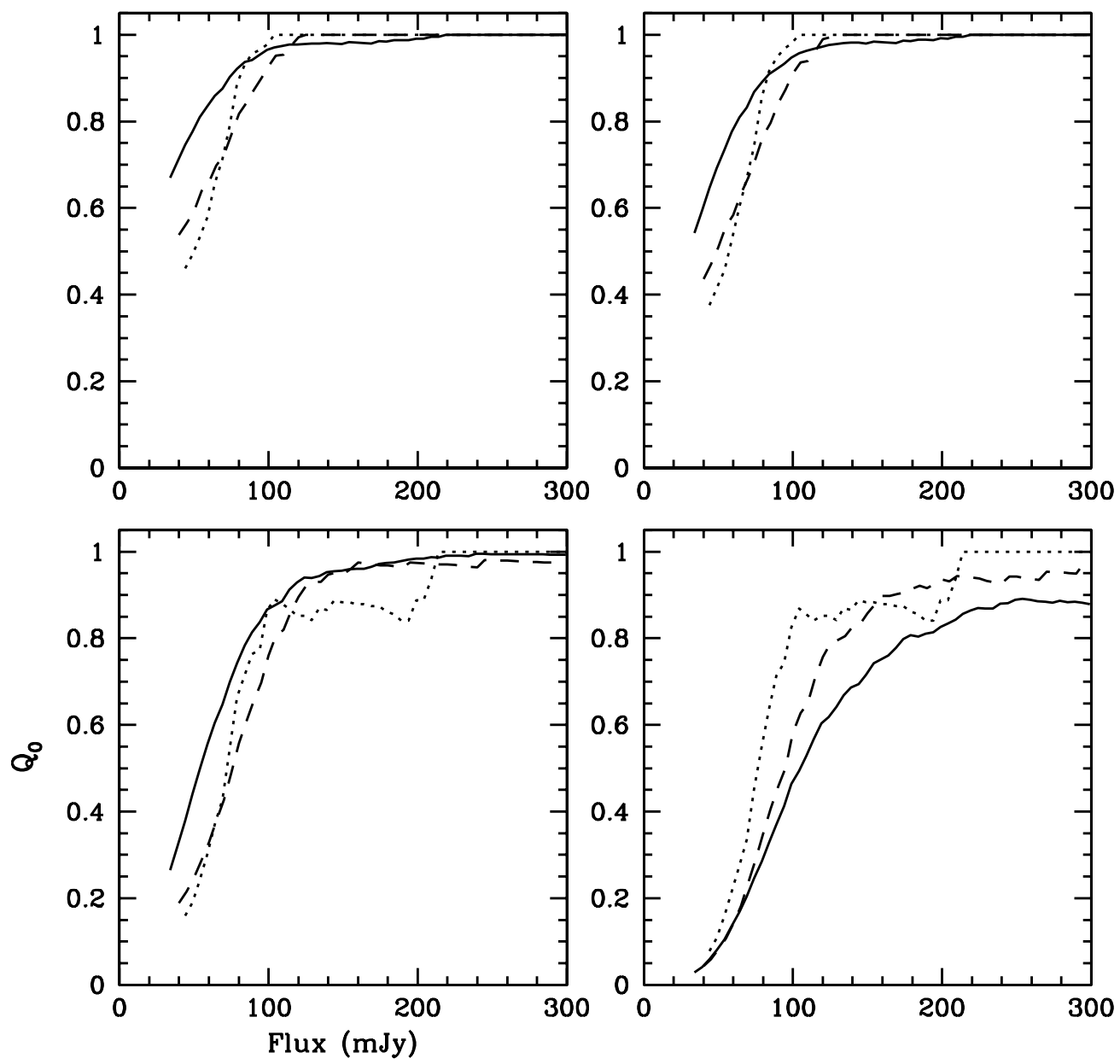

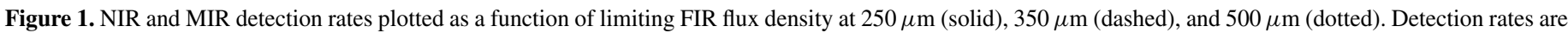

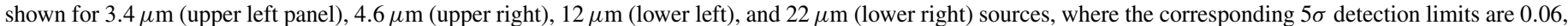

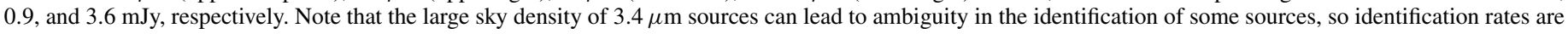
typically $\sim 10 \%-20 \%$ lower than the corresponding detection rates.

distribution, $f(r)$, the $3.4 \mu \mathrm{m}$ magnitude distribution of noncounterparts, $n(m)$, and the $3.4 \mu \mathrm{m}$ magnitude distribution of detected H-ATLAS counterparts, $q(m)$ :

$$
L=\frac{q(m) f(r)}{n(m)},
$$

where $f(r)$ is given by

$$
f(r)=\frac{1}{2 \pi \sigma_{\text {pos }}^{2}} \exp \left(-\frac{r^{2}}{2 \sigma_{\text {pos }}^{2}}\right) .
$$

We measure $n(m)$ directly from the WISE preliminary release catalog, while we determine $q(m)$ by first measuring the magnitude distribution of $3.4 \mu \mathrm{m}$ sources within $10^{\prime \prime}$ of the H-ATLAS source positions and then subtracting the magnitude distribution of background/foreground sources normalized by the area within this radius.

Following S11, we assume the astrometric uncertainty to be circularly symmetric and to have a dependence on the $250 \mu \mathrm{m}$ signal-to-noise ratio $(\mathrm{S} / \mathrm{N})$ given by,

$$
\sigma_{\text {pos }}=0.655 \frac{\text { FWHM }}{\mathrm{S} / \mathrm{N}},
$$

where FWHM $=18^{\prime \prime} .1$ is the Herschel beamwidth. While the WISE random astrometric uncertainties are negligible $\left(\sim 0^{\prime} .3\right)$, the WISE positions in the preliminary release catalog are known to suffer from highly non-Gaussian systematic offsets that can be as large as $1^{\prime \prime}$, so we apply a lower limit of $\sigma_{\text {pos }}>1^{\prime \prime} .5$.

Once we have computed the likelihood, $L_{j}$, for a possible counterpart $j$, we determine the reliability by summing over all possible counterparts,

$$
R_{j}=\frac{L_{j}}{\sum_{i} L_{i}+\left(1-Q_{0}\right)} .
$$

We consider any $3.4 \mu \mathrm{m}$ source with $R>0.8$ to be a reliable counterpart. After performing this procedure separately for WISE sources with star- and galaxy-like [3.4] - [4.6] colors, we obtain a combined total of 13,898 WISE counterparts, or $50.6 \% \pm 0.4 \%$ of the H-ATLAS catalog. The expected number of false matches can be obtained with

$$
N_{\text {falseID }}=\sum_{i}\left(1-R_{i}\right)
$$

We estimate that there are 369 false identifications in our sample, corresponding to a contamination rate of $2.7 \%$. The majority of our IDs have galaxy-like colors, but we were able to identify 14 objects with star-like colors $([3.4]-[4.6]<0)$ that were not already identified as galaxies with SDSS matching. This corresponds to an upper limit of $0.05 \%$ for the fraction of H-ATLAS sources identified as stars and detected in WISE. 


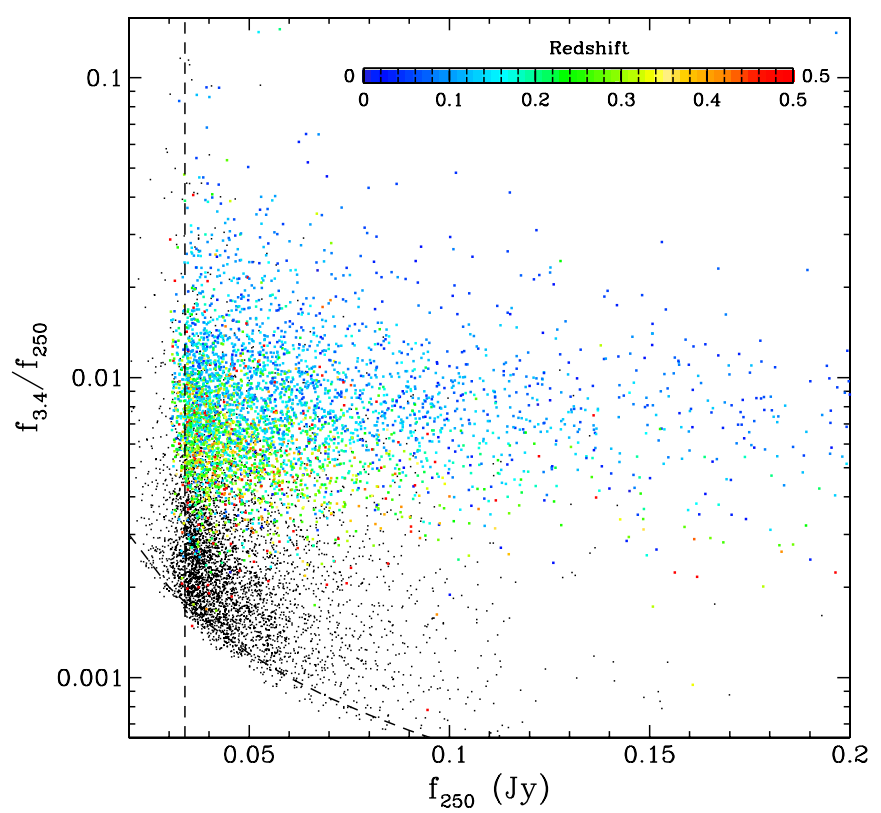

Figure 2. Distribution of the 3.4-250 $\mu \mathrm{m}$ flux density ratio as a function of $250 \mu \mathrm{m}$ flux density for H-ATLAS sources with WISE $3.4 \mu \mathrm{m}$ IDs. Colored points have spectroscopic redshifts from the GAMA survey and sources without measured redshifts are indicated by black points. Dashed lines indicate the approximate limits of the H-ATLAS and WISE surveys. The majority of sources with spectroscopic redshifts $(r \lesssim 19.4)$ are well detected by WISE, while the no-redshift sources have small flux density ratios that are inconsistent with "normal" low-redshift galaxies.

Comparing the individual WISE identifications to SDSS cross-identifications, we find that $84.7 \%$ of the 10,709 G15 H-ATLAS sources with $R>0.8$ SDSS identifications also had $R>0.8$ WISE identifications. The median separation between the WISE and SDSS sources is 0.'6, consistent with the astrometric uncertainties in the current version of the WISE catalog. In addition, $\sim 6 \%$ of these sources have separations $>3^{\prime \prime}$, consistent with the expected contamination rates of the WISE $(\sim 3 \%)$ and SDSS $(\sim 5 \%)$ matched samples.

\section{DEMOGRAPHICS}

The redshift distribution of $250 \mu \mathrm{m}$ selected sources is likely bimodal, with populations of both moderately star-forming galaxies at $z<1$ and high-redshift starburst galaxies at $z \sim 2$ (Smith et al. 2011; Lapi et al. 2011). Because the latter are typically faint in the observed-frame optical and UV, the vast majority of objects with measured spectroscopic or photometric redshifts are in the low-redshift mode. Of the H-ATLAS sources with spectroscopic and photometric redshifts (see the inset in Figure 3), we successfully identify $85.6 \%$ in WISE.

Although most of the WISE identifications with known redshifts are at $z<0.8(>98 \%)$, such a sample is biased toward low redshift because redshift measurements require detectable flux in the UV, optical, or near-infrared. Approximately one-third $(30.4 \%)$ of the identified sources have reliable spectroscopic redshift and two-thirds $(66.5 \%)$ have photometric redshifts from optical/NIR data. For the remaining sources, we can estimate redshifts using their FIR flux density ratios (e.g., Amblard et al. 2010; Schulz et al. 2010), which give an indication of the location of the peak of the FIR dust emission. In Figure 3, we compare the SPIRE colors of our matched catalog to a suite of $10^{6}$ modified blackbody SEDs (Amblard et al. 2010). We indicate on the plot the median flux density ratios of the 4677

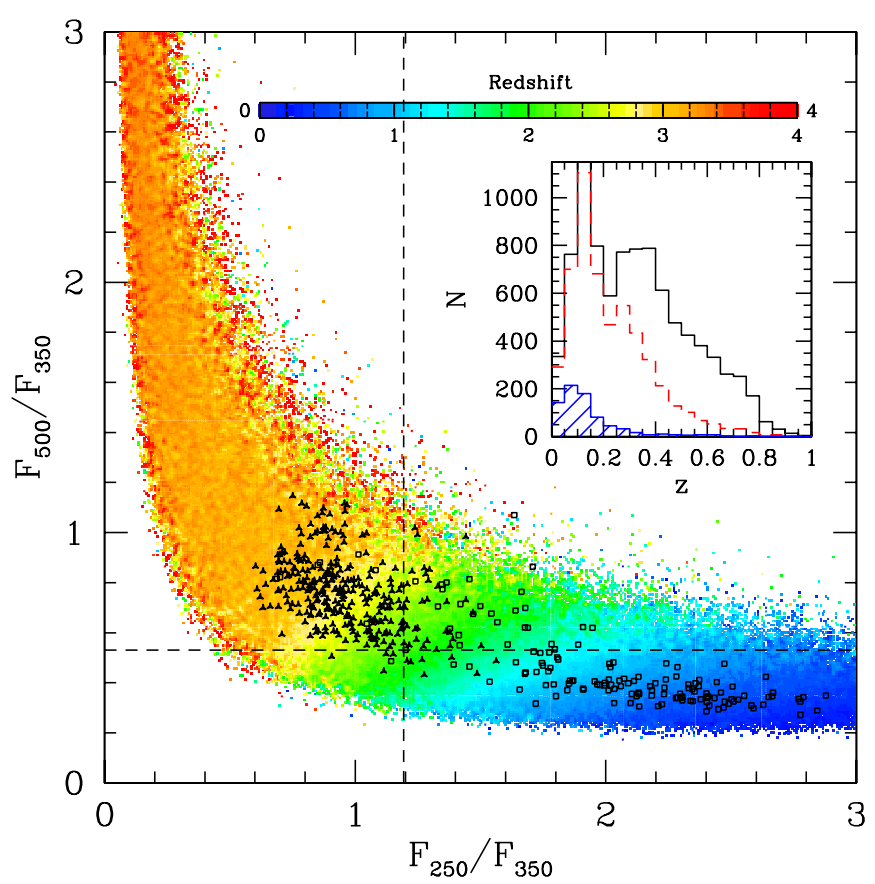

Figure 3. FIR flux density ratio for H-ATLAS sources with WISE identifications. Colored grid cells indicate the median redshift of a set of modified blackbody models with temperatures uniformly distributed between 10 and $60 \mathrm{~K}$ and emissivity parameters between zero and two. Black points indicate sources with $5 \sigma$ detections in all three SPIRE bands, where open squares have spectroscopic redshifts, $z<0.2$, and solid triangles have no spectroscopic or photometric redshift measurement. Dashed lines indicate the median flux density ratios of a stack of sources lacking redshifts. The majority of the no-redshift sources appear to be at $z \sim 2$. Inset: combined distribution of spectroscopic and photometric redshifts for H-ATLAS sources with detections at $3.4 \mu \mathrm{m}$ (solid histogram), $12 \mu \mathrm{m}$ (dashed), and $22 \mu \mathrm{m}$ (hatched). There are 73 sources at $z>1$ not shown.

H-ATLAS sources with WISE identifications but no redshifts ("no-redshift" sample, dashed lines), as well as the individual flux density ratios of 311 such sources with $>3 \sigma$ detections in all three SPIRE bands.

The no-redshift sample has median FIR colors suggesting $z \sim 2$, while the subset with three-band SPIRE detections may have even higher redshifts, perhaps as large as $z \sim$ 3.5. The redshifts derived using the Amblard et al. (2010) technique are highly uncertain, as redshift is degenerate with dust temperature and it is possible that these galaxies have typical dust temperatures different than $35 \mathrm{~K}$ (which is typical of high- $z$ submillimeter galaxies (SMGs); e.g., Chapman et al. 2005). However, in order for the no-redshift sample to be at $z \sim 0$, they would need to have very cold dust temperatures $(T \sim 12 \mathrm{~K})$ in addition to being very faint in the optical $(r \gtrsim 20.5)$. Although temperatures as low as $10 \mathrm{~K}$ have been seen in H-ATLAS galaxies at $z<1, T \sim 25-30 \mathrm{~K}$ is more typical (Dye et al. 2010). If the no-redshift sample has median temperatures typical of these low-redshift H-ATLAS galaxies, then our redshift estimate drops to $z \sim 1.5$.

Further evidence that the no-redshift sources are at $z \gg$ 0 can be found in the $3.4-250 \mu \mathrm{m}$ ratios (see Figure 2). H-ATLAS sources with GAMA redshifts have NIR-FIR flux density ratios that decrease from $\sim 0.009$ at $z \sim 0$ to $\sim 0.006$ at $z \sim 0.35$. This trend, which continues toward higher redshift, is due primarily to the larger $k$-correction at $3.5 \mu \mathrm{m}$ than at $250 \mu \mathrm{m}$ (e.g., Rieke et al. 2009). The median flux density ratio for the no-redshift sources, by comparison, is 0.0023 . Although low-redshift galaxies will occasionally exhibit such 


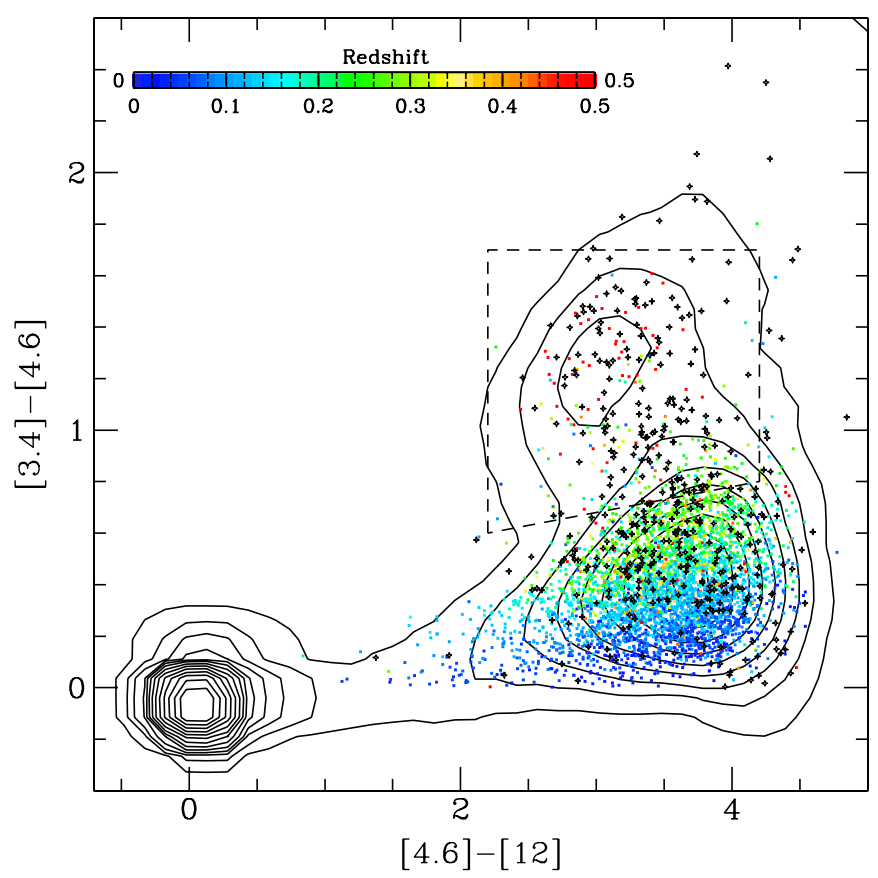

Figure 4. IR color distribution of ATLAS-WISE matches with three-band WISE photometry, including sources with $z<0.5$ (color coded by redshift) and noredshift sources (black points). The diagram can be divided into stars (lower left corner), star-forming galaxies (lower right corner), and AGNs (dashed region). The color distribution of all three-band WISE detections (including sources unmatched to H-ATLAS) is indicated by contours. Sources without measured redshifts tend to be redder in [3.4] - [4.6] and have a larger AGN fraction than sources with spectroscopic or photometric redshift measurements, $z<0.5$.

small NIR-FIR flux density ratios-Arp 220, for example, has $f_{3.4} / f_{250}=0.0019$ - such objects are rare, actively starforming, and unlikely to have dust temperatures as small as $\sim 15 \mathrm{~K}$.

We can shed even further light on the properties of the noredshift sample by examining their position in WISE color space (see Figure 4). The technique is similar to those developed for a set of IRAC filters at comparable wavelengths (Sajina et al. 2005; Stern et al. 2005). It was demonstrated that the diagram can be divided into stars and early-type galaxies (lower left corner, [4.6] $-[12] \lesssim 1$ ), star-forming galaxies (lower right corner), and active galactic nuclei (AGNs), where AGNs are selected largely based on their power-law emission in the NIR and MIR. This was adapted for WISE by Jarrett et al. (2011),

$$
\begin{aligned}
& {[4.6]-[12]>2.2} \\
& {[4.6]-[12]<4.2} \\
& {[3.4]-[4.6]<1.7} \\
& {[3.4]-[4.6]>0.1([4.6]-[12])+0.38}
\end{aligned}
$$

Of the 4959 H-ATLAS sources with WISE identifications, $z<0.5$, and detections in $3.4,4.6$, and $12 \mu \mathrm{m}$, the majority are star-forming galaxies, with $<0.2 \%$ main-sequence stars (which is consistent with the SDSS/H-ATLAS matching done by Thompson et al. 2010), and a small AGN fraction (0.057).

For the $9 \%$ of no-redshift sources with three-band WISE photometry, we find a much larger AGN fraction (0.30), but are subject to a selection bias toward AGNs due to their additional flux from warm dust at $12 \mu \mathrm{m}$ (Hainline et al. 2011). Furthermore, emission from polycyclic aromatic hydrocarbons in high-redshift star-forming galaxies can occasionally mimic AGNs in their IRAC colors, leading to contamination in our

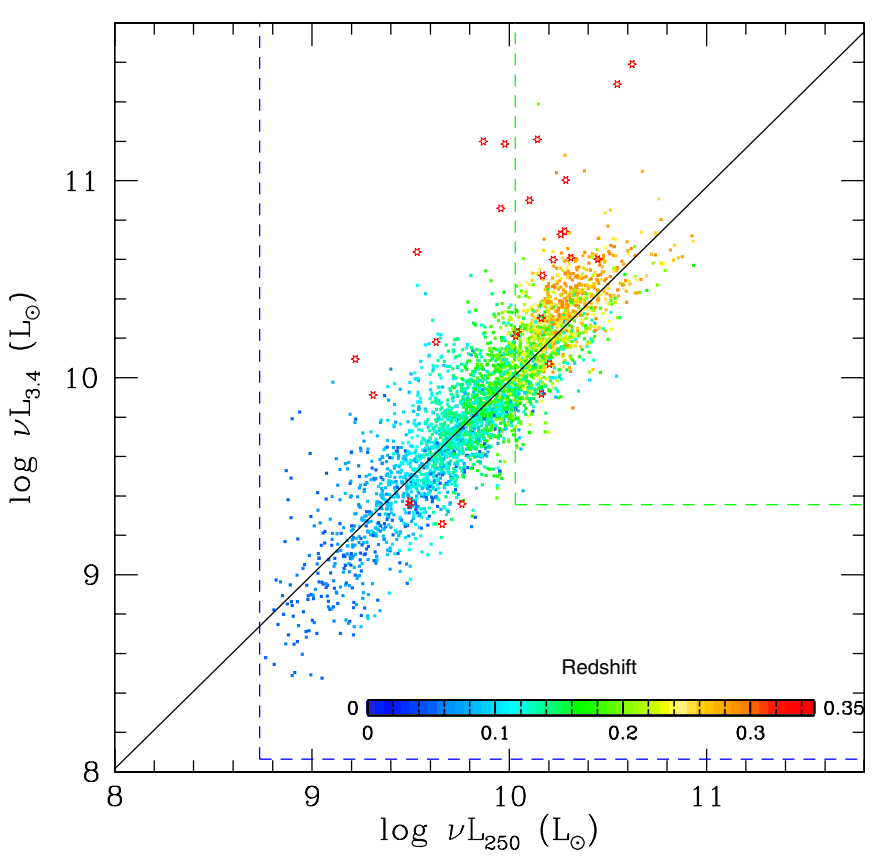

Figure 5. Luminosity at $3.4 \mu \mathrm{m}$ as a function of luminosity at $250 \mu \mathrm{m}$ for H-ATLAS sources with spectroscopic redshifts, $0.05<z<0.3$. Both luminosities are rest-frame $k$-corrected. Small squares are colored according to their redshift and red stars indicate AGNs, selected with the criteria given in Equation (7). Dashed lines indicate the approximate selection limits for sources at $z=0.05$ and 0.2 . The majority of the AGNs lie above the relationship, suggesting that $L_{3.4}$ contains a non-negligible contribution from hot dust emitting near the central black hole.

AGN samples (e.g., Lacy et al. 2004; Donley et al. 2012). For comparison, Coppin et al. (2010) performed SED fits on a set of blank-field SMG samples and estimated that $\sim 15 \%$ of SMGs are dominated by an AGN in the mid-infrared.

\section{FIR-NIR PROPERTIES}

Emission from dust in the FIR is often used as an indicator of the total star formation rate in galaxies (e.g., Kennicutt 1998), under the assumption that most of the dust heating is provided by young stars and that the star-forming regions are optically thick. The $250 \mu \mathrm{m}$ bandpass samples the cooler dust emission, which can arise from both star-forming clouds and diffuse regions in the interstellar medium (e.g., Eales et al. 2010b; Dunne et al. 2011). By contrast, $3.4 \mu \mathrm{m}$ emission is dominated by stellar sources and is a probe of the stellar mass for galaxies without a strong AGN contribution (e.g., Stern et al. 2005).

In Figure 5, we plot $3.4 \mu \mathrm{m}$ luminosity as a function of $250 \mu \mathrm{m}$ luminosity for H-ATLAS sources with spectroscopic redshifts, $0.05<z<0.3$. We $k$-correct $3.4 \mu \mathrm{m}$ using a powerlaw interpolation of the [3.4] - [4.6] color. For the $250 \mu \mathrm{m}$ flux densities, the majority of the galaxies in our sample do not have sufficient FIR flux density to obtain reliable dust temperatures, as is needed for a proper $k$-correction. Instead, we use the median dust temperature of $26 \mathrm{~K}$ found by Dye et al. (2010) using PACS and SPIRE data from the H-ATLAS SDP of 1346 $0.1<z<0.5$ sources. Although the $r<19.4$ limit of the GAMA spectroscopic survey restricts the range of luminosities that can appear on the plot, we estimate that GAMA misses only $\sim 5 \%$ of matched $z<0.3$ galaxies based upon the deeper $(r \lesssim 20.5)$ subsample of H-ATLAS galaxies with photometric redshifts. 
We find that the majority of the IR-color-selected AGNs (red points) lie above the relationship for star-forming galaxies, as $L_{3.4}$ will contain a non-negligible contribution from hot dust emitting near the central black hole in addition to emission from low-mass stars. However, if we exclude AGNs, we find an approximately linear correlation, with a best-fit power-law index, $\alpha=0.98_{-0.05}^{+0.03}$. The quoted systematic uncertainties on the power-law index were determined by allowing for a range of possible dust temperatures, $18<T<34 \mathrm{~K}$, when performing $250 \mu \mathrm{m} k$-corrections. The intrinsic scatter about this relationship is $0.18 \pm 0.01 \mathrm{dex}$, or $\sim 50 \%$.

The existence of a linear correlation between $L_{250}$ and $L_{3.4}$ across one and a half decades in luminosity suggests a close relationship between the cold dust probed by the FIR and the stellar mass probed by the NIR. Previous indications with Herschel have shown the $250 \mu \mathrm{m}$ luminosity density to tightly correlate with both the $24 \mu \mathrm{m}$ luminosity density, a star formation rate indicator, and the total infrared luminosity (Elbaz et al. 2010). This fact, coupled with a linear correlation between stellar mass and star formation rate for the general star-forming galaxy population (Daddi et al. 2007; Elbaz et al. 2007; Noeske et al. 2007; Donoso et al. 2012), suggests that the majority of the low-redshift H-ATLAS sources are actually "normal" starforming galaxies.

This publication makes use of data products from the Wide-field Infrared Survey Explorer, which is a joint project of the University of California, Los Angeles, and the Jet Propulsion Laboratory/California Institute of Technology, funded by the National Aeronautics and Space Administration.

Herschel is an ESA space observatory with science instruments provided by European-led Principal Investigator consortia with significant participation from NASA. U.S. participants in Herschel ATLAS acknowledge support provided by NASA through a contract issued from JPL.

In addition, we thank Dan Stern and Roberto Assef for helpful discussions about the quasar selection.

\section{REFERENCES}

Abazajian, K. N., Adelman-McCarthy, J. K., Agüeros, M. A., et al. 2009, ApJS, 182,543

Amblard, A., Cooray, A., Serra, P., et al. 2010, A\&A, 518, L9

Chapman, S. C., Blain, A. W., Smail, I., \& Ivison, R. J. 2005, ApJ, 622, 772

Coppin, K., Pope, A., Menéndez-Delmestre, K., et al. 2010, ApJ, 713, 503

Daddi, E., Dickinson, M., Morrison, G., et al. 2007, ApJ, 670, 156

Donley, J. L., Koekemoer, A. M., Brusa, M., et al. 2012, ApJ, 748, 142

Donoso, E., Yan, L., Tsai, C., et al. 2012, ApJ, 748, 80

Driver, S. P., Hill, D. T., Kelvin, L. S., et al. 2011, MNRAS, 413, 971

Dunne, L., Gomez, H. L., da Cunha, E., et al. 2011, MNRAS, 417, 1510

Dye, S., Dunne, L., Eales, S., et al. 2010, A\&A, 518, L10

Eales, S., Chapin, E. L., Devlin, M. J., et al. 2009, ApJ, 707, 1779

Eales, S., Dunne, L., Clements, D., et al. 2010a, PASP, 122, 499

Eales, S. A., Smith, M. W. L., Wilson, C. D., et al. 2010b, A\&A, 518, L62

Elbaz, D., Daddi, E., Le Borgne, D., et al. 2007, A\&A, 468, 33

Elbaz, D., Hwang, H. S., Magnelli, B., et al. 2010, A\&A, 518, L29

Fleuren, S., Sutherland, W., Dunne, D. J. B., et al. 2012, arXiv:1202.3891

Griffin, M. J., Abergel, A., Abreu, A., et al. 2010, A\&A, 518, L3

Hainline, L. J., Blain, A. W., Smail, I., et al. 2011, ApJ, 740, 96

Hopwood, R., Wardlow, J., Cooray, A., et al. 2011, ApJ, 728, L4

Jarrett, T. H., Cohen, M., Masci, F., et al. 2011, ApJ, 735, 112

Kennicutt, R. C., Jr. 1998, ApJ, 498, 541

Lacy, M., Storrie-Lombardi, L. J., Sajina, A., et al. 2004, ApJS, 154, 166

Lapi, A., González-Nuevo, J., Fan, L., et al. 2011, ApJ, 742, 24

Lawrence, A., Warren, S. J., Almaini, O., et al. 2007, MNRAS, 379, 1599

Negrello, M., Hopwood, R., De Zotti, G., et al. 2010, Science, 330, 800

Negrello, M., Perrotta, F., González-Nuevo, J., et al. 2007, MNRAS, 377, 1557

Noeske, K. G., Weiner, B. J., Faber, S. M., et al. 2007, ApJ, 660, L43

Pascale, E., Ade, P. A. R., Bock, J. J., et al. 2008, ApJ, 681, 400

Pilbratt, G. L., Riedinger, J. R., Passvogel, T., et al. 2010, A\&A, 518, L1

Poglitsch, A., Waelkens, C., Geis, N., et al. 2010, A\&A, 518, L2

Rieke, G. H., Alonso-Herrero, A., Weiner, B. J., et al. 2009, ApJ, 692, 556

Rigby, E. E., Maddox, S. J., Dunne, L., et al. 2011, MNRAS, 415, 2336

Sajina, A., Lacy, M., \& Scott, D. 2005, ApJ, 621, 256

Schulz, B., Pearson, C. P., Clements, D. L., et al. 2010, A\&A, 518, L32

Smith, D. J. B., Dunne, L., Maddox, S. J., et al. 2011, MNRAS, 416, 857

Spergel, D. N., Bean, R., Doré, O., et al. 2007, ApJS, 170, 377

Stern, D., Eisenhardt, P., Gorjian, V., et al. 2005, ApJ, 631, 163

Sutherland, W., \& Saunders, W. 1992, MNRAS, 259, 413

Thompson, M. A., Smith, D. J. B., Stevens, J. A., et al. 2010, A\&A, 518, L134

Wright, E. L., Eisenhardt, P. R. M., Mainzer, A. K., et al. 2010, AJ, 140, 1868 